\title{
Evidence of Pituitary Hypoplasia Associated with Partial Central Diabetes Insipidus in a Young Persian Cat
}

\author{
Emanuelle Bortolotto Degregori', Matheus da Rosa Pippi ${ }^{2}$, Ana Lúcia Ottolia Niederauer de Moura ${ }^{3}$, \\ Rogério Soila ${ }^{4}$, Priscila Viau Furtado ${ }^{4}$ \& Alan Gomes Pöpp ${ }^{2,5}$
}

Background: Congenital anomalies are an uncommon pituitary hypofunction cause associated to multiple hormone deficiencies. Congenital hyposomatotropism is often related to an inherited anomaly, characterized mainly by delayed growth. It is not uncommon to find associated thyroid-stimulating hormone and gonadotropin deficiencies. Pituitary malformation may be associated to progressive cystic lesion expansion. Central diabetes insipidus (CDI) is another rare disease associated to polyuria (PU) and polydipsia (PD) secondary to antidiuretic hormone (ADH) deficient secretion. The aim of this report is to describe a likely case of pituitary hypoplasia, associated with partial CDI in a cat.

Case: A 9-month-old unneutered male Persian cat weighing $2 \mathrm{~kg}$ was presented due to severe polyuria and polydipsia associated with growth deficit when compared with its sibling. After clinical and laboratory evaluations during the months in which the patient was monitored, reduced serum concentration of insulin-like growth factor-1 (IGF-1), thyroid-stimulating hormone (TSH), thyroid hormones, and testosterone were documented, confirming the diagnosis of hyposomatotropism, hypogonadism, and secondary hypothyroidism. Furthermore, therapeutic diagnosis with desmopressin revealed partial central diabetes insipidus (CDI). As the sibling showed normal development aging 13-months, a radiographic examination of the forelimb (carpus) was performed on both cats. There was lack of growth plate fusion in the patient, without any other evidence of dysgenesis, whereas complete epiphyseal closure was observed in the sibling. Despite therapeutic prescriptions of desmopressin and levothyroxine, the owners refused further follow-up to the case.

Discussion: Notwhistanding neuroimaging was not available for investigation of pituitary aspect in this particular case, the clinical symptoms added to the results of the complementary tests were consistent with pituitary hypoplasia, associated with hyposomatotropism, secondary hypothyroidism, hypogonadism, and partial CDI. Hyposomatotropism was presumably diagnosed based on the patient's clinical characteristics, which included proportional growth delay, delayed tooth eruption, delayed growth plate fusion, associated with serum reduced IGF-1 results in comparison with its sibling. The report of low free T4 by equilibrium dialysis and of low total T4 levels, associated with low TSH levels, was considered compatible with secondary hypothyroidism. TRH stimulation test is considered the gold standard for secondary hypothyroidism diagnosis since low TSH could be secondary to assay's low sensibility. However, normal TSH and thyroid hormone results in the sibling results ruled out this possible dismissed diagnose. The patient's lack of sexual interest, associated with hypotestosteronemia and underdeveloped genitals (absence of penile spines and testicular hypoplasia), indicates hypogonadism. Finally, partial CDI diagnosis was demonstrated by cat's partial ability to increase urinary specific gravity under water deprivation often made by the owners, as well as the response pattern to desmopressin therapy. Owing the lack of neurological signs expected to be associated with neoplastic or traumatic hypopituitarism etiology, hypoplasia hypothesis was raised. Quite often, patients with pituitary hypoplasia develop Rathke cleft cysts that might expand over time. In the present case, partial CDI was likely to be caused by the compression of the neurohypophysis by cystic formation secondary to adenohypophyseal hypoplasia since this kind of pituitary congenital anomaly does not justify per se neurohypophysis implications.

Keywords: hypopituitarism, hyposomatotropism, pituitary dwarfism, secondary hypothyroidism, polyuria and polydipsia. 


\section{INTRODUCTION}

Pituitary hypofunction is often related to tumors or traumas, but congenital anomalies have been seldom reported. Low production of more than one pituitary hormone characterize hypofunction $[3,7,11]$.

Hyposomatotropism is an endocrine disease resulting from primary growth hormone $(\mathrm{GH})$ and secondary insulin-like growth factor-1 (IGF-1) deficiencies. It is uncommon among dogs because of an inherited anomaly, and even more rare among cats, in which it is characterized mainly by delayed growth. It is not uncommon to find thyroid-stimulating hormone (TSH) and gonadotropin deficiencies associated with hyposomatotropism $[2,3,7,9]$.

Diabetes insipidus (DI) is a disease associated with severe polyuria (PU) and polydipsia (PD) secondary to a urinary concentrating defect. In cats, DI has been only described as central diabetes insipidus (CDI) because of defects in the synthesis, transport, or release of the antidiuretic hormone (ADH) $[1,10]$.

The aim of this study was to report a likely case of congenital pituitary hypoplasia in a young cat, resulting in hormone multi deficiency.

\section{CASE}

A 9-month-old unneutered male Persian cat weighing $2 \mathrm{~kg}$ presenting with severe PU/PD (water intake of $1.2 \mathrm{~L} / 24 \mathrm{~h}$ ) since the age of 3 months was brought into the Endocrinology Service. Growth deficit was clearly observed when compared to its sibling (Figure 1 A\&B). The patient had previous history of dermatophytosis and pneumonia, both of them previously treated (Table 1). On physical examination, the patient was active, with no cognitive deficit, dehydration level of 5-7\%, and no evidence of goiter or traumas, despite dental malocclusion (Figure 1C).
Its clinical parameters were in accordance with the reference standards for the species.

Blood samples were collected for serum biochemical tests (Table 1). Serum hormone's concentrations were assessed in the patient and in its sibling, allowing for comparisons between them (Table 2). Levels lower than the reference range for total thyroxine (TT4) and TSH were documented for the patient, indicating secondary hypothyroidism, whereas the test results were normal for its sibling. Regarding IGF-1, the sibling showed levels greater than the reference range, as expected for animals during the growth stage, while the patient had IGF-1 levels lower than $50 \%$ of the concentration observed in its sibling (Table 2). GH measurements before and after a stimulation test were not performed for not being commercially available.

Abdominal ultrasound was performed to assess possible differential diagnoses, but no noteworthy findings were obtained. Urinalysis revealed isosthenuria (Table 1). In the additional tests and in the clinical picture, there were grounds for suspecting of partial CDI associated with dwarfism and congenital hypothyroidism.

The water deprivation test was not considered for confirmation of DI because of its side effects and because the owners had been restricting water intake prior to the each veterinary visit with the purpose of reducing PU and preventing voiding incidents on their way to the clinic (located more than $200 \mathrm{~km}$ away from their place of residence). A computed tomography (CT) or magnetic resonance (MR) scan was recommended to investigate the possible etiology of hyposomatotropism and CDI, ruling out possible previous trauma or neoplasm. However, since the owners were reluctant to grant authorization, neuroimaging was not performed.

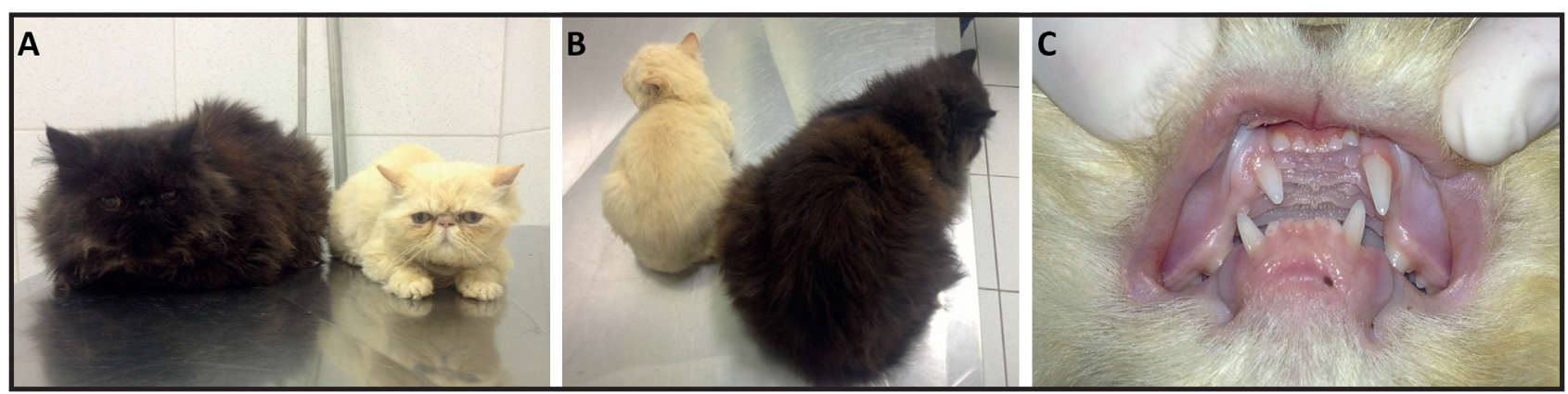

Figure 1. A- Patient's frontal view (cream coat cat) with growth deficit as compared to its sibling (black coat cat) at 9-months of age. B- Siblings' dorsal view further showing growth deficit. C- Delayed tooth eruption and malocclusion. 
Table 1. Hematologic, serum biochemistry, and urine analysis results during patient's follow-up.

\begin{tabular}{|c|c|c|c|c|c|c|}
\hline & 8 Мо.* & 9 Mo.** & 12 Mo. & 13 Mo. & 14 Mo. & Reference \\
\hline Erythrocytes $\left(\mathrm{x} 10^{3} / \mathrm{mm}^{3}\right)$ & 8.08 & - & 10.24 & - & 4.92 & $5-10.5$ \\
\hline Hemoglobin (g/dL) & 13.4 & - & 13.5 & - & 11.4 & $8-15$ \\
\hline Hematocrit (\%) & 41 & - & 41 & - & 35 & $24-45$ \\
\hline Leukocytes $\left(\mathrm{x} 10^{6} / \mathrm{mm}^{3}\right)$ & 23.3 & - & 14 & - & 15 & $5-19.5$ \\
\hline Neutrophils $\left(\mathrm{x} 10^{6} / \mathrm{mm}^{3}\right)$ & 18.7 & - & 6.16 & - & 7.65 & $2.5-12.5$ \\
\hline Lymphocytes (x10\%/mm) & 4.3 & - & 4.6 & - & 5.1 & $1.5-7$ \\
\hline TPP (g/L) & 82 & - & 74 & - & 62 & $60-80$ \\
\hline Albumin $(\mathrm{g} / \mathrm{L})$ & 32 & - & 36 & - & 34 & $21-33$ \\
\hline ALT (U/L) & 97 & 80 & 39 & 41 & 48 & $<83$ \\
\hline $\mathrm{ALP}(\mathrm{U} / \mathrm{L})$ & 215 & 343 & 176 & 35 & - & $<93$ \\
\hline Creatinine (mg/dL) & 1.02 & 0.93 & 1.3 & 1.21 & 1.01 & $0.8-1.8$ \\
\hline Urea (mg/dL) & - & 82 & 32 & 61.7 & 49 & $32-54$ \\
\hline Phosphorus (mg/dL) & - & 10.5 & 5.1 & 7.96 & 7.7 & $2.7-6.2$ \\
\hline Cholesterol (mg/dL) & 129 & - & 116 & 113.4 & - & $95-130$ \\
\hline Glucose (mg/dL) & 72 & - & 96 & 105 & 85 & $73-134$ \\
\hline Triglycerides (mg/dL) & - & - & 35 & 29.2 & - & $25-133$ \\
\hline Fructosamine & 215 & - & 247 & - & - & $219-247$ \\
\hline Venous plasma $\mathrm{pH}$ & - & 7.38 & 7.26 & - & 7.36 & $7.27-7.4$ \\
\hline Sodium (mEq/L) & - & $>180$ & 174 & 170.2 & 160 & $145-157$ \\
\hline Potassium (mEq/L) & - & - & 3.6 & 4.5 & 4 & $3.6-5.5$ \\
\hline $\mathrm{iCa}(\mathrm{mmol} / \mathrm{L})$ & - & 1.16 & 1.29 & - & 1.32 & $1.07-1.5$ \\
\hline Serum osmolality (mOsm/kg) & - & 371 & 376 & 377 & 350 & $280-310$ \\
\hline Urine specific gravity & 1,018 & 1,014 & 1,008 & - & 1,004 & $>1,035$ \\
\hline Urine $\mathrm{pH}$ & 6.0 & 6.0 & 6.0 & - & 6.0 & $6.5-7.5$ \\
\hline UP/UC ratio & 0 & 0 & 0.5 & - & 0 & $<0.5$ \\
\hline
\end{tabular}

Mo: months; *: first visit: treatment of dermatophytosis and pneumonia; **: first visit to the Endocrinology Service; TPP: total plasmatic proteins; ALT: alanine transaminase; ALP: alkaline phosphatase; iCa: ionized calcium; UP/UC: urinary protein to urinary creatinine ratio; <: lower; >: higher.

Three months after the first visit (at the age of 12 months), the patient showed weight gain $(0.5 \mathrm{~kg})$, but it had no sexual interest, whereas its sibling was sexually active. Physical examination revealed small testicles, penile hypoplasia, and absence of penile spines. Serum hormones (Table 2), complete blood count, serum biochemical parameters, and urine (Table 1) were then reassessed. Serum TT4 and TSH levels were lower than in the previous test. Moreover, free T4 levels measured by dialysis and testosterone levels were also lower than the reference range for the species. Based on those findings, hypogonadism was diagnosed, and secondary hypothyroidism was considered confirmed.
Thyrotropin-releasing hormone (TRH) test for confirmation of secondary hypothyroidism was not performed because of the risks and prohibitive price to owners.

Four months after the first visit (at the age of 13 months), given the urinalysis results (Table 1), the desmopressin (DDAVP) test was performed, and one drop/day $(1.5 \mu \mathrm{g} /$ day $)$ of intranasal DDAVP ${ }^{1}$ was initially instilled into the patient's conjunctival sac. When reassessed 1 week after daily DDAVP administration, the patient showed lower water intake and less frequent urination, as reported by the owners, but only on the first days of treatment. The owners also reported the patient had had ad libitum access to water prior to the 
Table 2. Hormone concentration of both cats at 9 months and of the patient at 12 months.

\begin{tabular}{ccccc}
\hline & $\begin{array}{c}\text { Patient at } \\
\text { months }\end{array}$ & $\begin{array}{c}\text { Sibling at } 9 \\
\text { months }\end{array}$ & $\begin{array}{c}\text { Patient at 12 } \\
\text { months }\end{array}$ & Reference range \\
\hline TT4 $(\mu \mathrm{g} / \mathrm{dL})$ & 0.96 & 1.88 & 0.41 & $1.5-4.0$ \\
FDT4 $(\mathrm{ng} / \mathrm{dL})$ & 1.15 & 2.07 & 0.55 & $0.76-3.94$ \\
TT3 $(\mathrm{ng} / \mathrm{mL})$ & 0.41 & 0.76 & - & $0.3-1.5$ \\
TSH $(\mathrm{ng} / \mathrm{mL})$ & 0.03 & 0.14 & 0.02 & $0.05-0.50$ \\
IGF-1 $(\mathrm{nmol} / \mathrm{L})$ & 44 & 96 & 48 & $12-92$ \\
Insulin $(\mathrm{uU} / \mathrm{mL})$ & - & - & 7.85 & $4-15$ \\
Testosterone $(\mathrm{ng} / \mathrm{mL})$ & - & - & 0.16 & $1-6$ \\
\hline
\end{tabular}

TT4: total thyroxine; FDT4: free thyroxine by equilibrium dialysis; TT3: total triiodothyronine. All hormones were measured by radioimmunoassay, except for TSH, which was determined using chemiluminescence.

visit. New hematological, serum biochemistry, and urine analysis were requested (Table 1). Despite the low urine specific gravity, increased plasma osmolality was observed. The DDAVP dose was adjusted to one drop every $12 \mathrm{~h}(3 \mu \mathrm{g} / \mathrm{day})$, and levothyroxine ${ }^{2}$ therapy at a dose of $50 \mu \mathrm{g} / \mathrm{day}$ was recommended for hypothyroidism treatment.

While sibling was showing normal development, the patient was showing some growth, despite a clear deficit was still evident (Figure 2 A\&B). A radiographic examination of the right forelimb (carpus) was performed in both cats. There was lack of growth plate fusion in the patient, without any other evidence of dysgenesis (Figure 2C), compatible with hyposomatotropism, whereas complete epiphyseal closure (Figure 2D) was observed in the sibling. Unfortunately, the owners did not bring the patient for the scheduled reassessments (loss to follow-up). The owners reported by e-mail the cat never reached PU/PD satisfactory control and was always sleepy. At age of 5-years it was found dead in the pool, probably by accident while drinking water.

\section{DISCUSSION}

The case reported herein has similar findings to those observed in cats with pituitary hyposomatotropism and partial CDI, probably resulting from pituitary hypoplasia. This condition leads to insufficient synthesis of hormones secreted by the adenohypophysis (GH, FSH, LH, and TSH) and by the neurohypophysis (ADH) $[1-3,7,11,12]$.

Pituitary dwarfism because of a genetic mutation in Lhx3 gene is infrequent among dogs, and it is even more rare among cats $[2,5,11,12]$. In the present case report, hyposomatotropism was presumably diagnosed based on the patient's clinical characteristics, which included proportional growth delay, delayed tooth eruption, delayed growth plate fusion, and hypogonadism, in line with what has been described by Reed \& Gunn-Moore [9].

The patient was referred to the Endocrinology Service at the age of nine months, but the owners had already observed signs of linear growth delay since the patient was three months old, when compared to its sibling, a fact that concurs with the literature, according to which growth problems become evident around the 3 rd to the 5 th months of life $[2,3,5,9]$.

The patient's IGF-1 levels were within the reference range for the species, but lower than those observed in its sibling, indicating probable GH partial deficiency. Another characteristic of this particular case that argues in favor of partial deficiency is the fact that the patient showed discrete growth over time, despite still underdeveloped contrasting with its sibling. According to Reed \& Gunn-Moore [9], kittens usually have IGF-1 levels above the reference range because of their growth. Moreover, the patient's growth was delayed when compared to their siblings, which was confirmed by the lack of epiphyseal closure, as indicated in the literature $[3,5,9,11,12]$.

The patient's lack of sexual interest, associated with hypotestosteronemia and underdeveloped genitals (absence of penile spines and testicular hypoplasia), indicates hypogonadism, corroborating the hypothesis of pituitary hypoplasia. In addition, in hypogonadism observed in pituitary dwarfism, cryptorchidism and azoospermia can also be present. However, some animals might have normal reproductive functions [9]. 


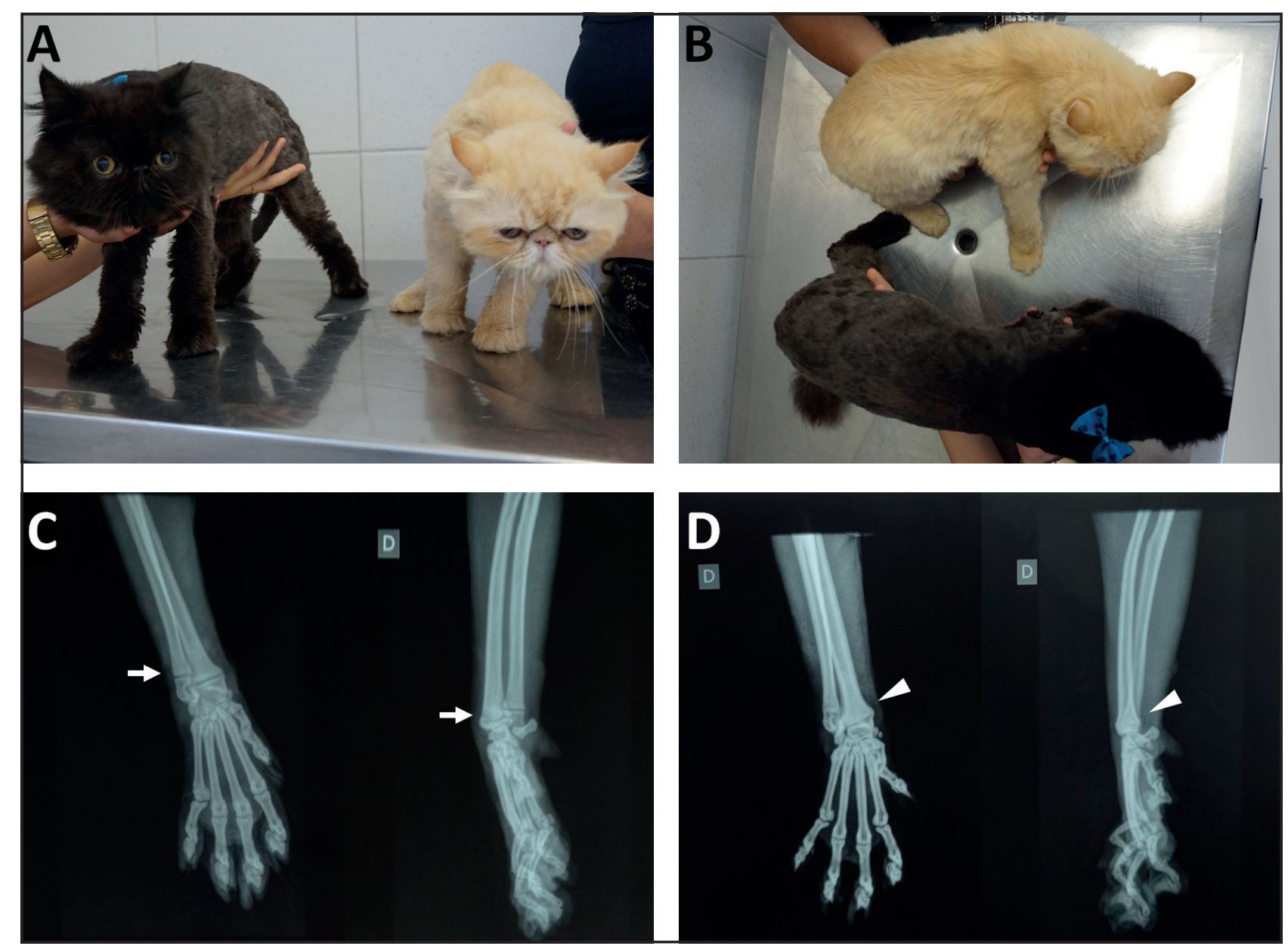

Figure 2. A- Patient's frontal view (cream coat cat) with growth deficit as compared to its sibling (black coat cat) at 13-months of age. B- Siblings' dorsal view further showing growth deficit. C- Radiographic image of right forelimb (carpus) of the patient at 13 months showing lack of growth plate fusion (white arrows). D- Complete epiphyseal closure in the sibling at the same age (white arrow heads).

Even though there are no commercial kits for GH measurements in cats that allow performing the GH stimulation test for confirmation of hyposomatotropism [5], the diagnosis of pituitary dwarfism can be made based on the patient's clinical symptoms and on the fact that the results of complementary tests rule out other conditions that co-occur with growth deficit and low IGF-1 levels, as pointed out by Voorbij et al. [12]. Hormone replacement therapy for hyposomatotropism, however, has not been safe and efficacious in cats [5]. Given the absence of autologous $\mathrm{GH}$, in addition to the fact that progestogen therapy causes considerable side effects in cats and does not lead to satisfactory growth, hormone replacement therapy was not recommended for hyposomatotropism in this case.

The report of low free $\mathrm{T} 4$ by equilibrium dialysis and of low total T4 levels, associated with low TSH levels, is compatible with secondary hypothyroidism. Confirmation of secondary hypothyroidism would depend on the TRH stimulation test [9], which was not performed because of its prohibitive cost and risks. Nevertheless, considering the TSH results within the reference range for the species in the sibling, the possibility that the lack of sensitivity of the TSH assay could have been the cause for low TSH concentration was ruled out. Therefore, the difference in TSH values between the patient and its sibling, the abnormal radiographic findings, and the slightly disproportionate appearance observed over time indicate possible chronic thyroid dysfunction.

The patient had severe PU and PD, with a water intake of $1.2 \mathrm{~L} /$ day $(600 \mathrm{~mL} / \mathrm{kg} / \mathrm{day})$ at initial presentation. Urine output greater than $50 \mathrm{~mL} / \mathrm{kg}$ /day and water intake larger than $100 \mathrm{~mL} / \mathrm{kg} /$ day are common manifestations of CDI and, quite often, they are the only clinical signs observed $[6,10]$. However, since the patient had PU and PD, which are also compatible with other conditions, complementary tests were requested 
to rule out other PU/PD causes. CDI patients could have other clinical signs such as urinary incontinence, loss of appetite, and weight loss [10]. Those signs, however, were not described by the owners, and even though CDI patients prefer water to solid food, the patient investigated herein showed normal appetite, subtle growth, and weight gain.

During the first assessments, the patient was kept by the owners on a water-restricted diet to prevent it from urinating in inappropriate places, since specialized veterinary treatment was only available miles away, and that might have led to hyperosmolality and urine concentration increase from hyposthenuria to isosthenuria. Hyposthenuria was observed only when the patient received ad libitum access to water. These findings are compatible with partial CDI, in which, despite restricted access to water, urine specific gravity does not exceed 1,015 to 1,020 , even if the patient is dehydrated $[3,10]$.

Water deprivation and desmopressin tests are used for the diagnosis of CDI [1,6]. As the owners opposed the water deprivation test and as this test is currently less indicated because of its risks, it was not performed, and the desmopressin test was then recommended. Notwithstanding the initial response on the first days of DDAVP treatment, with reduction of water intake and of urine output, and just a small reduction in plasma osmolality, there was recommendation for increasing the desmopressin dosage from 1 to 2 daily doses because of hyposthenuric urine specific gravity. The control of CDI in cats is attained with one to three drops of DDAVP twice or three times a day, but the response to the therapy may vary [10]. Also, desmopressin response may occur on an individual basis and the doses should be adjusted according to the resolution of the clinical signs. It is possible that the owners themselves may have adjusted the doses and obtained a more satisfactory control of CDI, prompting them not to bring the patient back for the follow-up visits. The administration of intranasal DDAVP in the conjunctival sac is well established and considered to be appropriate as continuous therapy in cats [6]. Nonetheless, when hormone replacement therapy is not possible, the animal should always receive ad libitum access to water [10].

A limitation of this case report is the failure to assess pituitary gland images using CT, MRI, or post-mortem examination, not allowing for the confirmation of the underlying cause of hyposomatotropism, hypothyroidism, hypogonadism, and CDI. Since there is partial deficiency of several adenohypophyseal hormones, the clinical and laboratory evidence gathered in this case report allows suggesting that pituitary hypoplasia could be the potential cause. Advanced imaging diagnostic techniques, such as MRI and CT, would be reliable for assessing the etiology of CDI in cases of neoplasms, cysts, trauma, or pituitary hypoplasia $[1,8]$. However, owing to the lack of neurological signs, which are associated with a neoplastic or traumatic etiology, and because of the reduced likelihood of neoplasms in young cats, this possibility was ruled out.

Quite often, patients with pituitary hypoplasia develop Rathke cleft cysts that might expand over time [9]. In the present case, partial CDI was likely to be caused by the compression of the neurohypophysis by cystic formation secondary to adenohypophyseal hypoplasia since this kind of pituitary congenital anomaly does not justify per se neurohypophysis implications.

The prognosis of cases with multiple hormone deficiencies (TSH, IGF-1, and GH) is poor and, in general, these patients will eventually die at a young age [11]. Nevertheless, when only acquired or congenital CDI is present, the animals may become asymptomatic and have a superior quality of life after receiving appropriate therapy and care [10]. Unfortunately, because of the loss to follow-up and loss of contact with the owners, long-term follow-up and therapeutic adjustments were not possible.

This report concludes even in absence of a more advanced neuroimaging method, that clinical evidence combined with complementary exams results, indicates the presence of hyposomatotropism, secondary hypothyroidism, and hypogonadism. These findings suggest a possible adenohypophyseal hypoplasia, with associated partial CDI. However, the cause of pituitary hypofunction could not be detected.

\section{MANUFACTURERS}

${ }^{1}$ Laboratório Químico Farmacêutico Bergamo. Taboão da Serra, SP, Brazil.

${ }^{2}$ Abbott Laboratórios do Brasil Ltda. São Paulo, SP, Brazil.

Funding. The present report was supported by the Coordenação de Aperfeiçoamento de Pessoal de Nível Superior (CAPES), Brazil. Código de Financiamento 001.

Acknowledgements. The authors would like to thank to the PROVET. Medicina Diagnóstica for their support to conduct this study.

Declaration of interest. The authors report no conflicts of interest. The authors alone are responsible for the content and writing of paper. 


\section{REFERENCES}

1 Aroch I., Mazaki-Tovi M., Shemesh O., Sarfaty H. \& Segev L. 2005. Central diabetes insipidus in five cats: clinical presentation, diagnosis and oral desmopressin therapy. Journal of Feline Medicine and Surgery. 7(6): 333-339.

2 Donaldson D., Billson F.N. \& Scase T.J. 2008. Congenital hyposomatotropism in a domestic shorthair cat presenting with congenital corneal oedema. Journal of Small Animal Practice. 49(6): 306-309.

3 Greco D.S. 2012. Pituitary Deficiencies. Topics in Companion Animal Medicine. 27(1): 2-7.

4 Gunn-Moore D. 2013. Hypothyroidism in cats. In: Rand J. (Ed). Clinical Endocrinology of Domestic Animals. Ames: Wiley-Blackwell, pp.273-277.

5 König M.L., Henke D., Adamik K. \& Vera C.P. 2018. Juvenile hyposomatotropism in a Somali cat presenting with seizures due to intermittent hypoglycaemia. Journal of Feline Medicine and Surgery Open Report. 4(1): 1-6.

6 Medeiros L.K.G., Carneiro R.S., Alves A.S., Mendes R.S. \& Nóbrega Neto P.I. 2014. Central diabetes insipidus in a cat. Acta Scientiae Veterinariae. 42(51): 1-4.

7 Meij B.P., Voorhout G., Gerritsen R.J., Grinwis G.C. \& Ijzer J. 2012. Lymphocytic hypophysitis in a dog with diabetes insipidus. Journal of Comparative Pathology. 147(4): 503-507.

8 Oliveira K.M., Fukushima F.B., Oliveira C.M., Rosado I.R., Torres B.B.J., Lavor M.S.L., Silva C.M.O. \& Melo E.G. 2012. Head trauma as a possible cause of central diabetes insipidus in a cat. Journal of Feline Medicine and Surgery. 15(2): 155-159.

9 Reed N. \& Gunn-Moore D. 2013. Hyposomatotropism in cats. In: Rand J. (Ed). Clinical Endocrinology of Domestic Animals. Ames: Wiley-Blackwell, pp.416-420.

10 Reed N. \& Gunn-Moore D. 2013. Diabetes insipidus in cats. In: Rand J. (Ed). Clinical Endocrinology of Domestic Animals. Ames: Wiley-Blackwell, pp.450-457.

11 Silvestrini P., Piviani M. \& Ruiz de Gopegui R. 2008. Enanismo hipofisario congénito en un gato siamés. Clínica Veterinaria de Pequeños Animales. 28(3): 169-172.

12 Voorbij A.M., Van S.F.G., Vos-Loohuis M., Martens E.E., Hanson-Nilsson J.M., Van-Oost B.A., Kooistra H.S. \& Leegwater P.A. 2011. A contracted DNA repeat in $L H X 3$ intron 5 is associated with aberrant splicing and pituitary dwarfism in German shepherd dogs. PLoS One. 6(11): e27940. 